CORRECTION

\title{
Correction: Genetic and clinical aspects of Wolfram syndrome 1 , a severe neurodegenerative disease
}

\author{
Luciana Rigoli ${ }^{1}$, Placido Bramanti ${ }^{2}$, Chiara Di Bella ${ }^{1}$ and Filippo De Luca ${ }^{1}$ \\ Pediatric Research (2018) 84:787; https://doi.org/10.1038/s41390-018-0146-1
}

Correction to: Pediatric Research; https://doi.org/10.1038/pr.2018.17; published online 28 Feb 2018

The original version of this article erroneously cropped part of the abstract. The abstract has now been corrected to read 'Wolfram syndrome 1 (WS1) is a rare autosomal recessive neurodegenerative disease characterized by diabetes insipidus, diabetes mellitus, optic atrophy, deafness, and other abnormalities. WS1 usually results in death before the age of 50 years. The pathogenesis of WS1 is ascribed to mutations of human WFS1 gene on chromosome $4 p$ encoding a transmembrane protein called wolframin, which has physiological functions in membrane trafficking, secretion, processing, and/or regulation of ER calcium homeostasis. Different types of WFS1 mutations have been identified, and some of these have been associated with a dominant, severe type of WS. Mutations of CISD2 gene cause autosomal recessive Wolfram syndrome 2 (WS2) characterized by the absence of diabetes insipidus and psychiatric disorders, and by bleeding upper intestinal ulcer and defective platelet aggregation. Other WFS1-related disorders such as DFNA6/14/38 nonsyndromic low-frequency sensorineural hearing loss and Wolfram syndrome-like disease with autosomal dominant transmission have been described. WS1 is a devastating disease for the patients and their families. Thus, early diagnosis is imperative to enable proper prognostication, prevent complications, and reduce the transmission to further progeny. Although there is currently no effective therapy, potential new drugs have been introduced, attempting to improve the progression of this fatal disease." in both the PDF and HTML versions of the article.

\footnotetext{
'Department of Pediatrics, University Hospital, Messina, Italy and ${ }^{2}$ RCCS Centro Neurolesi "Bonino-Pulejo", Messina, Italy Correspondence: Luciana Rigoli (luciana.rigoli@unime.it)
}

Published online: 31 August 2018 